\title{
Current Status of NIFS-SWJTU Joint Project for Quasi-Axisymmetric Stellarator CFQS*)
}

\author{
Mitsutaka ISOBE ${ }^{1,2)}$, Akihiro SHIMIZU ${ }^{1)}$, Haifeng LIU $^{3)}$, Hai LIU ${ }^{3)}$, Guozhen XIONG ${ }^{3)}$, \\ Dapeng YIN ${ }^{4)}$, Kunihiro OGAWA ${ }^{1,2)}$, Yasuo YOSHIMURA ${ }^{1)}$, Motoki NAKATA ${ }^{1,2)}$,

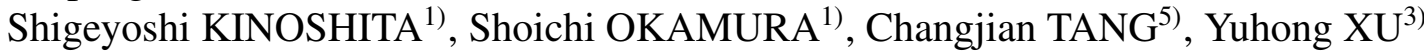 \\ and the CFQS Team ${ }^{1,2,3,4,5)}$ \\ ${ }^{1)}$ National Institute for Fusion Science, National Institutes of Natural Sciences, Toki 509-5292, Japan \\ ${ }^{2}$ SOKENDAI (The Graduate University for Advanced Studies), Toki 509-5292, Japan \\ ${ }^{3)}$ Institute of Fusion Science, School of Physical Science and Technology, Southwest Jiaotong University, \\ Chengdu 610031, People's Republic of China \\ 4) Hefei Keye Electro Physical Equipment Manufacturing Co., Ltd, Hefei 230000, People's Republic of China \\ ${ }^{5)}$ College of Physical Science and Technology, Sichuan University, Chengdu 610041, People's Republic of China
}

(Received 10 January 2019 / Accepted 28 February 2019)

The world's newest quasi-axisymmetric stellarator CFQS is constructed as a joint project of the National Institute for Fusion Science, Japan and Southwest Jiaotong University, the People's Republic of China. The project is named "NSJP for CFQS". The CFQS has a major radius of $1 \mathrm{~m}$, toroidal periods of 2, and aspect ratio of 4. The toroidal magnetic field strength will be increased up to $1 \mathrm{~T}$. The CFQS equilibrium is characterized by low-aspect ratio and quasi-axisymmetry, having both natures of two-dimensional tokamak and three-dimensional stellarator. The CFQS offers new opportunities and fields to increase understanding of toroidal fusion plasmas.

(C) 2019 The Japan Society of Plasma Science and Nuclear Fusion Research

Keywords: CFQS, CHS, CHS-qa, quasi-axisymmetric stellarator, effective helical ripple, optimized configuration, modular coil

DOI: $10.1585 /$ pfr. 14.3402074

\section{Introduction}

In recent years, helical plasma research has progressed greatly. The deuterium operation of the Large Helical Device (LHD) begun in March, 2017 [1-4] to explore a higher-performance helical plasma and the first plasma in Wendelstein 7-X was initiated in December, 2015 [5, 6]. Because a helical plasma based on three-dimensionality is very flexible in a magnetic configuration, continuous efforts to explore advanced configurations must be made steadily.

A quasi-axisymmetric stellarator is characterized by a tokamak-like axisymmetric magnetic field in the magnetic coordinates as the name suggests [7], and is attractive in terms of having no requirement for inductive plasma current, reduced neoclassical transport, low-toroidal viscosity, magnetic well in an entire region of plasma, and compactness through low-aspect-ratio characteristics. In addition, the quasi-axisymmetric stellarator offers the drift reversal capability of trapped particles due to weak nonaxisymmetric magnetic field components that can potentially suppress micro-instabilities driven by anomalous transport [8]. Physics and engineering designs of the

author'se-mail: isobe@nifs.ac.jp

*) This article is based on the presentation at the 27th International Toki Conference (ITC27) \& the 13th Asia Pacific Plasma Theory Conference (APPTC2018)
Compact Helical System (CHS)-qa as a post-CHS [9-13] and the National Compact Stellarator Experiment (NCSX) [14-17] based on a quasi-axisymmetry concept were intensively carried out around 2000 in the National Institute for Fusion Science (NIFS), Japan and the Princeton Plasma Physics Laboratory, United States, respectively. Also, the Evolutive Stellarator of Lorraine (ESTELL) was proposed later in France $[18,19]$. However, the experimental device was not realized. In order to explore the potential of expansion of helical plasma confinement, NIFS and Southwest Jiaotong University (SWJTU) in the People's Republic of China have agreed upon construction of the Chinese First Quasi-axisymmetric Stellarator (CFQS) with laboratory size but advanced configuration as a joint project.

\section{NIFS-SWJTU Joint Project for CFQS}

NIFS is contributing to the world stellarator program with remarkable experimental results from the LHD [1-4] covering various physical and engineering topics in fusion science and development. In addition to such contributions, NIFS started a new stellarator program in 2017 for a small size experiment with the purpose of exploring a high degree of freedom of three-dimensional magnetic configuration design, which is a great advantage for stellarators compared to tokamaks. NIFS and SWJTU have entered 


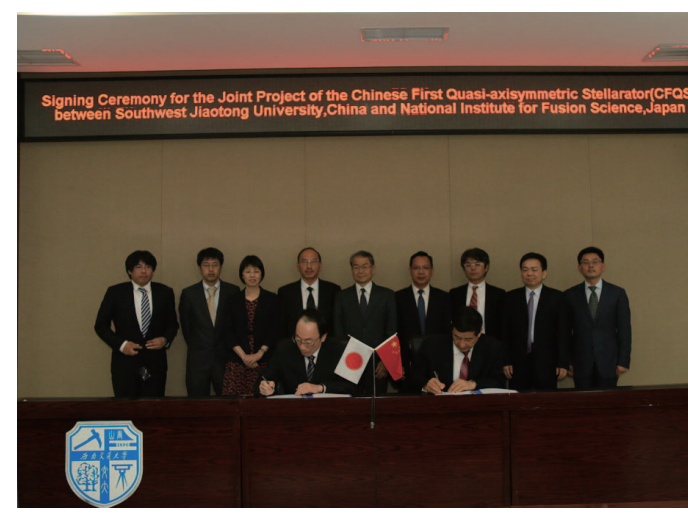

Fig. 1 Signing ceremony for the joint project of the CFQS between NIFS, Japan and SWJTU, China on July 3, 2017.

into an agreement for international academic cooperation to promote cooperative research in helical fusion plasma research. The signing ceremony was held on July 3, 2017, at SWJTU, as shown in Fig. 1. NIFS and SWJTU, together with implementing a joint project to design and construct a new helical device based upon a quasi-axisymmetry concept, will develop active academic interaction among researchers and students at both institutes.

NIFS and SWJTU, while receiving powerful support from the People's Republic of China's fusion research community, will implement design and construction, plasma heating and diagnostics technical development, and plasma experiments, and will introduce the helical device to be called CFQS. These two institutes also will initiate collaborative projects and rigorously cooperate in those projects. NIFS and SWJTU have organized the steering committee for the CFQS that will manage this joint project. This committee consists of members of NIFS and SWJTU and the steering committee meeting (SCM) is held regularly. The steering committee organizes subsidiary task forces, and these task forces will discuss specific topics, such as physics, engineering, heating, diagnostics, and other matters.

Responsibilities of NIFS and SWJTU are as follows. SWJTU prepares the experiment building, basic components of the CFQS, such as magnetic field coils, vacuum chamber, power supply, pure water cooling system, and other components necessary for the CFQS operation at SWJTU. NIFS provides the basic design of the CFQS, such as magnetic field configuration, modular coil geometry, and other design matters, based on experience in physics and engineering design of the CHS-qa [9-13]. NIFS also provides experts to support the equipment design and machine construction, and utilizes as many unused CHS apparatuses as possible for the CFQS. These include vacuum pumping system, plasma heating system, advanced diagnostics, such as $54.5 \mathrm{GHz}$ gyrotron of which maximum power is $\sim 450 \mathrm{~kW}$ with pulse duration of $100 \mathrm{~ms}$, a neutral beam injector $(\mathrm{NBI})$ of which acceleration voltage $\left(E_{N B}\right)$ and injection power $\left(P_{N B}\right)$ are $30 \mathrm{kV}$ and $1 \mathrm{MW}$, respec- tively, and heavy ion beam probe (HIBP).

\section{Design of CFQS}

Physics design of the CFQS was intensively performed as a collaborative work between NIFS and SWJTU in 2017. Fundamental properties of the CFQS, such as Fourier spectrum of magnetic field strength, mod-B contour, and profiles of rotational transform and magnetic well depth are available in Refs. 20-22. A study of feasible divertor based on magnetic island concept is also ongoing [23]. Primary device parameters of the CFQS were decided on May 30, 2018, through discussion in the SCM of the NIFS-SWJTU joint project as follows: major radius $(R)$ of $1 \mathrm{~m}$, number of toroidal periods $(N)$ of 2 , aspect ratio $\left(A_{p}\right)$ of 4 , maximum toroidal magnetic field strength $\left(B_{t}\right)$ of $1 \mathrm{~T}$, and 16 modular coils made of water-cooled hollow copper conductors. Although the CHS-qa was designed with $A_{p}$ of 3.2, we chose $A_{p}$ of 4 for the CFQS to avoid difficult technical challenges in the machine design and construction. Engineering design, for example, evaluation of electromagnetic force for each coil, design of support structure, vacuum vessel, power supply system, and other issues, are now being performed at NIFS, Hefei Keye Electro Physical Equipment Manufacturing Co., Ltd. (Keye), and SWJTU. Fabrication of a mock-up of the modular coil will be initiated at Keye in early 2019. Three-dimensional computer-aided drawing (3D CAD) of 16 modular coils and the last closed magnetic flux surface of CFQS are shown in Fig. 2.

The CFQS engineering design, i.e., modular coils, vacuum vessel (VV), diagnostics port arrangement on the $\mathrm{VV}$, and support structure against electromagnetic force are intensively being performed jointly with the help of the 3D CAD code CATIA, computer aided engineering code ANSYS based on finite element method, and a small 3D printer to check feasibility of machine construction [24]. In addition, arrangement of experiment building and design for the power supply and water cooling systems are ongoing. The complete view of CFQS equipped with a NBI of CHS is shown in Fig. 3.

In the CFQS, substantial amount of spontaneous bootstrap current is expected in high- $\beta$ regime, as discussed for the CHS-qa $[25,26]$, because of quasi-axisymmetry of the system. Effect of neoclassical bootstrap current (BSC) on MHD equilibrium of CFQS is investigated by using the BOOTSJ code, which calculates bootstrap currents in non-axisymmetric magnetic configurations using the drift kinetic equation [27]. Figures 4a) and 4b) show radial profiles of neoclassical BSC density $(d I / d s)$ in volumeaveraged $\beta$ of $0.59 \%$ as an example and rotational transforms for various $\beta$, respectively. Here, $s, I$, and $\beta$ represent normalized toroidal flux, value of $\mathrm{BSC}$, and value of volume-averaged beta, respectively. In this analysis, the MHD equilibrium is obtained with the VMEC code [28] in the free-boundary condition, and parabolic electron density and temperature profiles are assumed. As can 

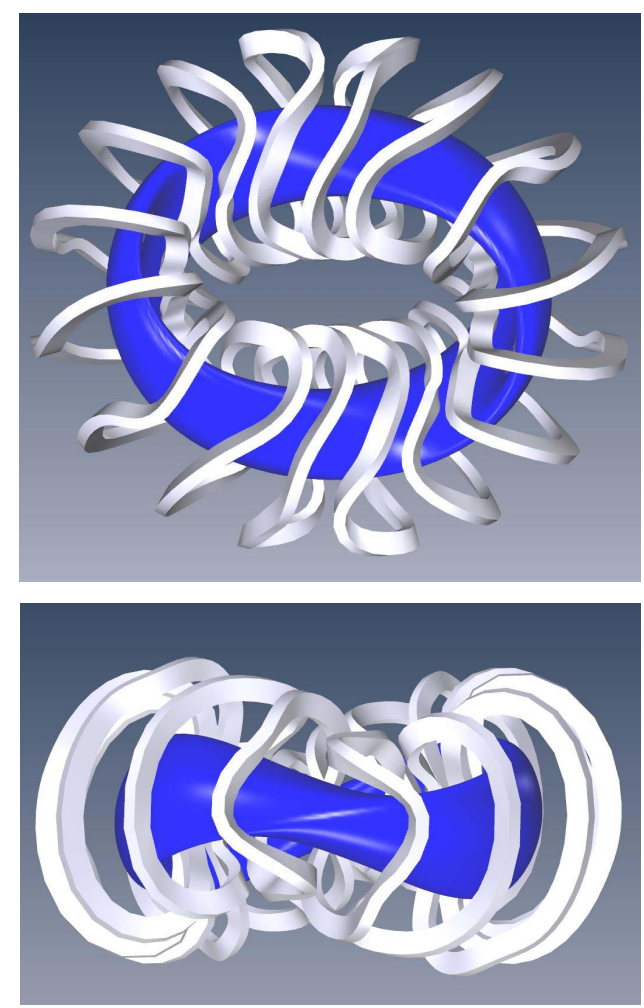

Fig. 2 Three-dimensional computer-aided drawing of 16 modular coils and the last closed magnetic flux surface of CFQS. The CFQS has a major radius of $1 \mathrm{~m}$, aspect ratio of 4 , and toroidal periods of 2 . The toroidal magnetic field strength will be increased up to $1 \mathrm{~T}$.

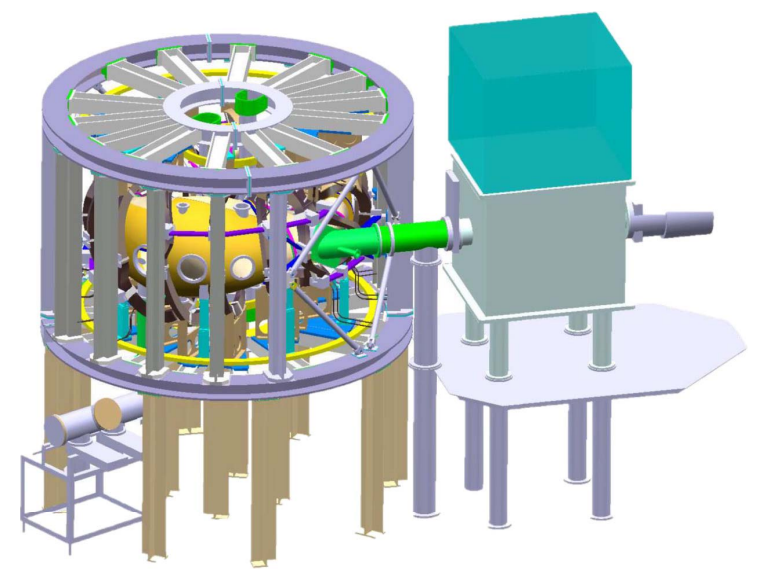

Fig. 3 Complete view of the CFQS equipped with a neutral beam injector of CHS. The major radius of CFQS plasma is $1 \mathrm{~m}$.

be seen, the rotational transform is significantly affected due to neoclassical BSC as $\beta$ increases. Because quasiaxisymmetry is realized in the CFQS, improvement of neoclassical transport is our great concern. In this work, we use the NEO code based on the field line integration technique to evaluate effective helical ripple modulation amplitude $\left(\varepsilon_{e f f}\right)$, which represents the effect of the magnetic field geometry upon $1 / v$ transport [29]. As can be seen in
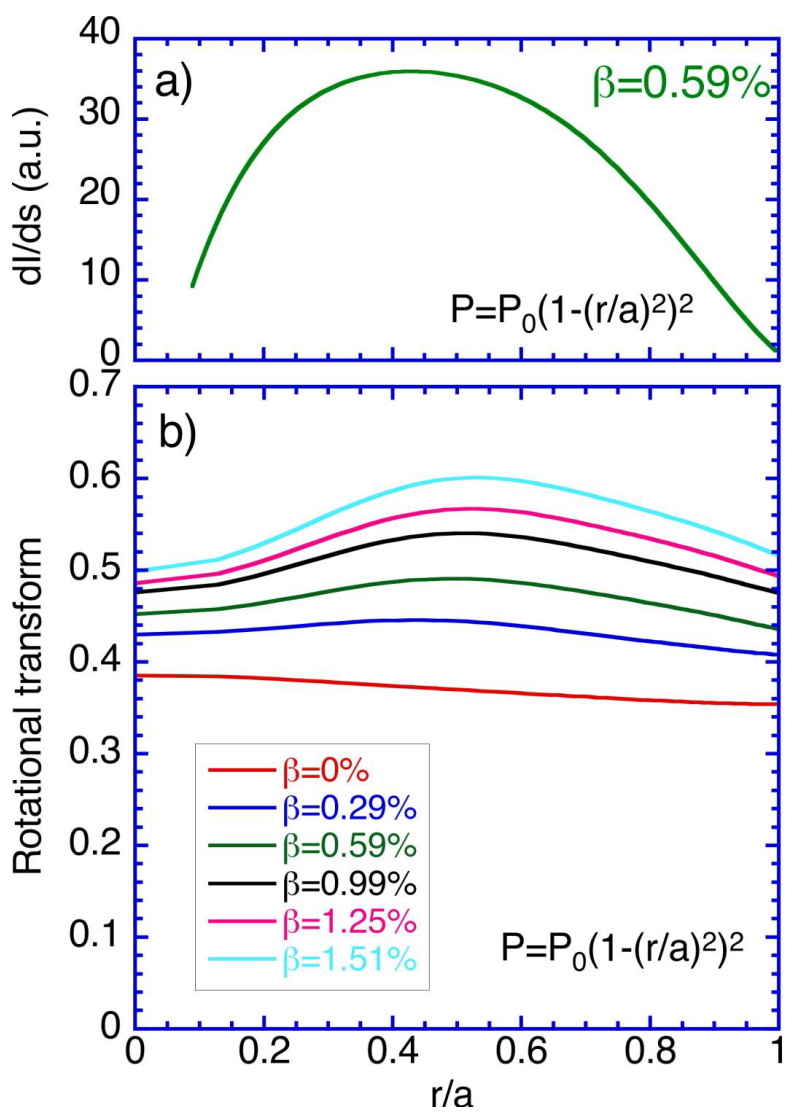

Fig. 4 Radial profiles of a) density of neoclassical bootstrap current in volume-averaged $\beta$ of $0.59 \%$ as an example, and b) rotational transform for various volume-averaged $\beta$. The pressure profile is assumed to be $P=P_{0}\left(1-(r / a)^{2}\right)^{2}$ in this analysis.

Fig. 5, $\varepsilon_{\text {eff }}$ in the CFQS equilibrium is significantly diminished, in particular, in the peripheral domain, compared with that of the CHS. Note that the CHS is a conventional helical device characterized by low $A_{p}$ of $4.5 \sim 5.0$, having the major radius of $1 \mathrm{~m}$, the multipolarity of 2 , and the toroidal periods of 8 [30]. Effect of finite $\beta$ with neoclassical BSC on $\varepsilon_{e f f}$ is also investigated for the CFQS. The favorable quasi-axisymmetry characteristics can be sustained even if $\beta$ increases in the CFQS configuration. In addition to fundamental physics properties of the CFQS mentioned above, recently, efforts to reveal micro instability, turbulent transport, and zonal flow generation characteristics for the CFQS equilibrium have been initiated by using the Gyrokinetic Vlasov code GKV [31].

\section{Plasma Heating and Diagnostics 4.1 ECRH/ECCD}

Electron cyclotron resonance heating (ECRH) has been used in helical device experiments to initiate a plasma and explore confinement property of high electron temperature $\left(T_{e}\right)$ plasmas in low-collisional or collisionless regimes. The CHS was equipped with two different gyrotrons for ECRH of which frequencies are $54.5 \mathrm{GHz}$ and 


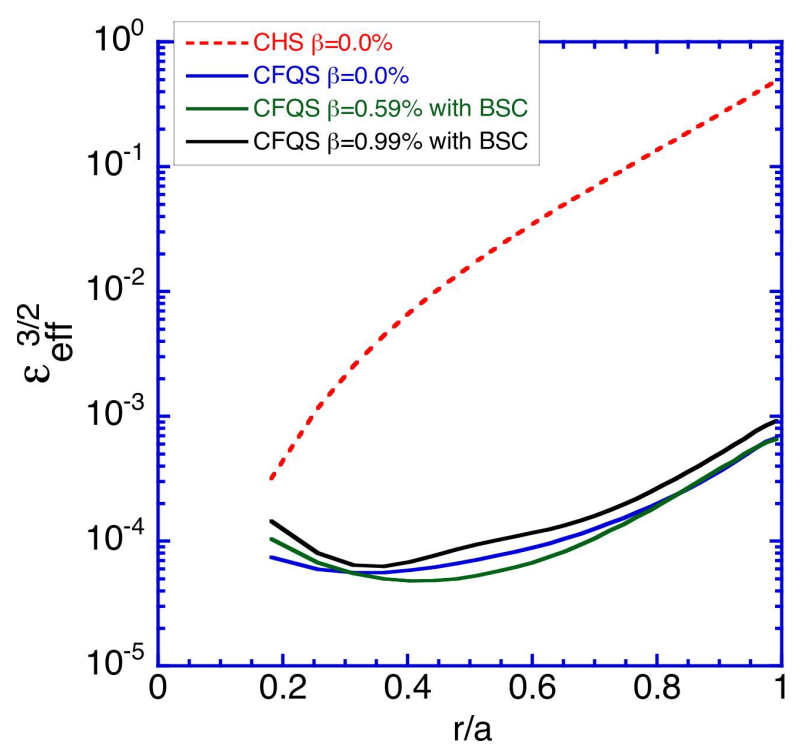

Fig. 5 Effective helical ripple as a function of normalized minor radius $(r / a)$ for CHS and CFQS MHD equilibria. In the finite $\beta$ cases, neoclassical bootstrap current is considered.

106.4 GHz [32]. Because the maximum $B_{t}$ of the CFQS is chosen to be $1 \mathrm{~T}$, the $54.5 \mathrm{GHz}$ gyrotron will be used for the CFQS. Note that magnetic field strength for second harmonic resonance $\left(B_{r e s}\right)$ is $0.974 \mathrm{~T}$. The maximum injection power $P_{E C R H}$ and pulse length of the $54.5 \mathrm{GHz}$ waves are $\sim 450 \mathrm{~kW}$ and $100 \mathrm{~ms}$, respectively.

Because the CFQS rotational transform is characterized by weak magnetic shear and is sensitive to $\beta$ value as shown in Fig. 4, a control of rotational transform will be extremely important. Electron cyclotron current drive (ECCD) can be one of the options for adjusting the rotational transform and will play an important role in CFQS experiment. The ECRH deposition in a CFQS plasma is evaluated by the ray tracing code TRAVIS [33]. Ray of electron cyclotron (EC) wave of $54.5 \mathrm{GHz}$ and second harmonic resonance layer for the EC wave are shown in Fig. 6. The EC wave is obliquely injected in toroidal direction and $B_{t}$ is adjusted to achieve central heating. In the CFQS, the EC wave will be launched from the midplane of the outboard side of the torus at the bean-shaped poloidal cross section. Radial profiles of electron density $\left(n_{e}\right)$ and $T_{e}$ assumed in this analysis are shown in Figs. 7 (a) and 7 (b), respectively. Radial profiles of deposited power density and EC-driven current density predicted by the TRAVIS code are shown in Figs. 7 (c) and 7 (d), respectively. Note that $P_{E C R H}$ is set to be $1 \mathrm{MW}$ in this calculation. Since a net current due to ECCD is proportional to the absorbed power of EC wave, a realistic ECCD current can be deduced from this analysis. The TRAVIS code suggests that total absorption power efficiency over $98 \%$ can be achieved in the core region and ECCD total current efficiency is fairly high, $229 \mathrm{kA} / \mathrm{MW}$. Referring to the results on relation between magnitude of net plasma current and modification of rota-

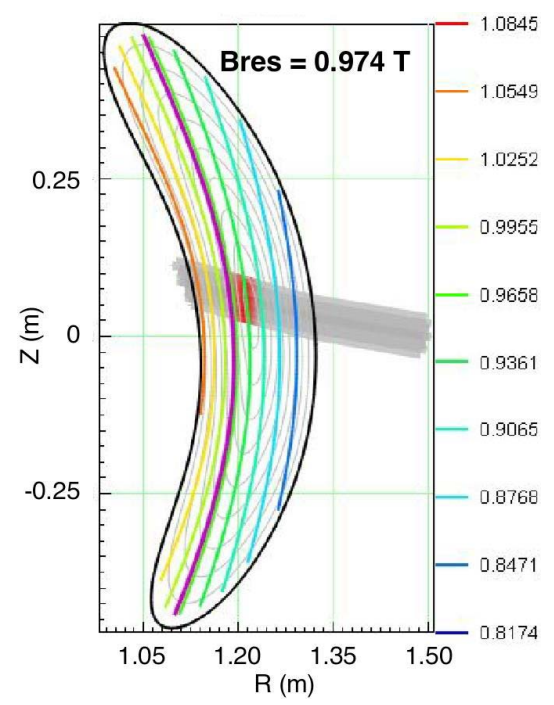

Fig. 6 Mod-B contours of CFQS at $B_{t}$ of $0.92 \mathrm{~T}$ and electron cyclotron resonance wave of $54.5 \mathrm{GHz}$ launched from the outboard side at the bean-shaped poloidal cross section. Each color line corresponds to different magnetic field strength.
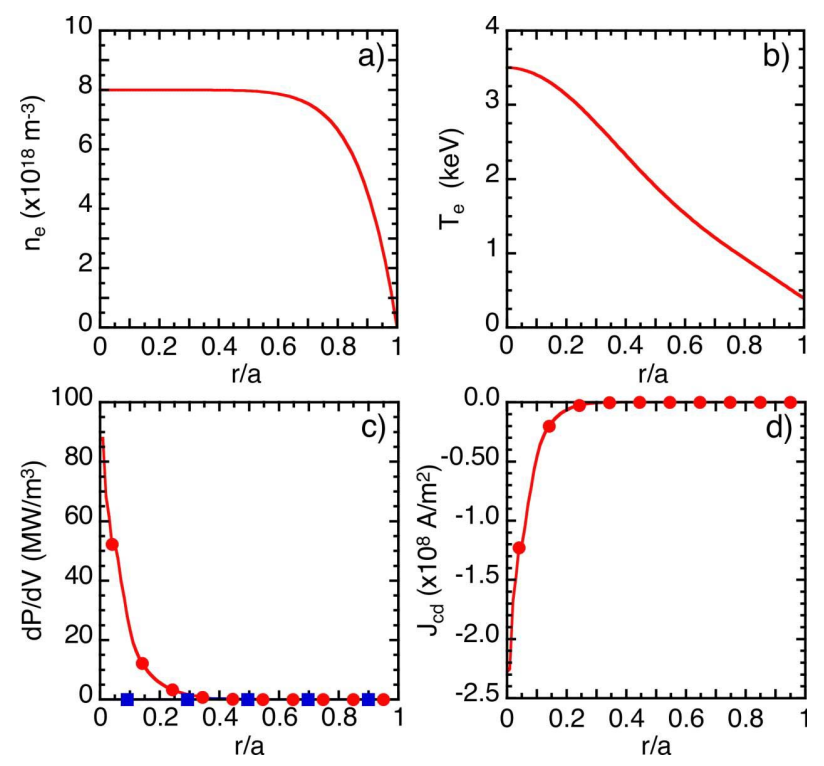

Fig. 7 Input plasma parameters and results calculated by the TRAVIS code. Radial profiles of a) electron density, b) electron temperature, c) deposited power density, and d) current density driven by ECCD. The injection power of electron cyclotron resonance wave of $54.5 \mathrm{GHz}$ is assumed to be $1 \mathrm{MW}$. In Fig. 7 (c), red circles and blue squares represent $d P / d V$ for passing electrons and trapped electrons, respectively. Here, $V$ stands for volume.

tional transform for the CHS-qa $[25,26]$, ECCD will be effective in the active adjustment of the rotational transform of the CFQS.

\subsection{NBI}

NBI is essentially required to realize a high- 


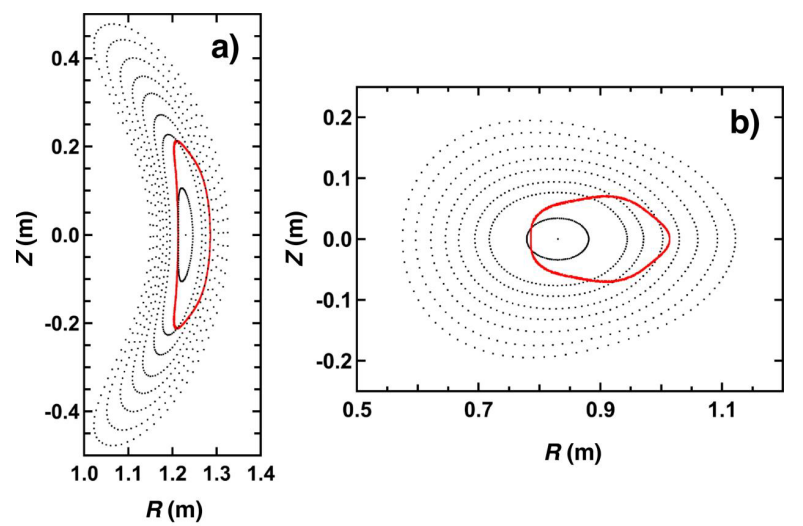

Fig. 8 Collisionless co-going transit beam ion orbit at two different toroidal angles in the CFQS equilibrium at $B_{t}$ of $1 \mathrm{~T}$. The orbit is calculated in the guiding center approximation. An energetic proton of which energy and pitch angle are $30 \mathrm{keV}$ and 22 degrees, respectively, is launched at the equatorial plane of the outboard side of torus.

temperature plasma and/or access high- $\beta$ regime. The CHS was equipped with two intense NBIs. NBIs in CHS played an important role in the achievement of high- $\beta[34,35]$ and high stored energy [36], and in physics studies of the edge transport barrier [37,38]. The beam line (BL) \#2 of CHS is relatively new and will be exported to SWJTU. The BL\#2 can deliver $P_{N B}$ of $1 \mathrm{MW}$ with $E_{b}$ of $30 \mathrm{kV}$.

In order to verify confinement of neutral beam (NB)injected energetic ions in the CFQS equilibrium, collisionless guiding center orbit calculation was performed by using the DELTA5D code [39]. Figure 8 shows collisionless co-going transit beam ion orbits at two different toroidal angles in the CFQS equilibrium at $B_{t}$ of $1 \mathrm{~T}$. An energetic proton of which energy and pitch angle are $30 \mathrm{keV}$ and 22 degrees, respectively, is launched at the outboard side of torus in this analysis. Note that beam ion velocity is nearly parallel to the magnetic field in this case because NB will be tangentially injected in the CFQS. As can be seen in Fig. 8, tangentially injected energetic beam ion provided by CHS BL\#2 can be confined well in the CFQS. More detailed analysis of confinement properties of beam ions and heating efficiency of NBI for the CFQS is ongoing [40].

\subsection{Diagnostics}

The CFQS plasma experiment will begin with fundamental diagnostics. The $2 \mathrm{~mm}$ microwave interferometer used in CHS will be employed to measure $n_{e}$ in the early phase of experiment. Because this interferometer will not work in high-density plasmas due to significant refraction of the microwave, a multi-channel interferometer based on a semiconductor characterized by high power and stability will be developed.

Magnetics, i.e., 1) Minov coil set for measurement of magnetic fluctuation and identification of mode numbers of MHD instabilities, 2) Rogowski coil to measure a net plasma current, such as a current induced by ECCD,
NBCD, and/or spontaneous bootstrap current, and 3) diamagnetic loop to evaluate plasma stored energy will be installed from the initial phase of experiment. In addition, the CFQS will have spectroscopic diagnostics such as $\mathrm{H} \alpha$ and vacuum ultraviolet diagnostics, and diagnostics to reveal edge plasma phenomenon such as Langmuir probe.

The CFQS is given status of proof-of-principle experiments because the CFQS is the first quasi-axisymmetric stellarator in the world. In the CFQS, confinement improvement due to low-toroidal viscosity leading to high toroidal rotation with rotation shear, suppression of turbulent transport by shear flow, and/or zonal flow generation characteristics will be primary physics targets. Therefore, the HIBP will play an important role in revealing physics above mentioned. In this project, the HIBP used in CHS [41-43] will be employed.

\section{Summary}

NIFS and SWJTU have entered into an agreement for international academic cooperation to promote cooperative research in helical fusion plasma research. Physics design of CFQS is almost completed. Engineering design is being performed jointly. The major parameters of the CFQS were decided to be $R$ of $1 \mathrm{~m}, A_{p}$ of $4, N$ of 2 , and maximum $B_{t}$ of $1 \mathrm{~T}$. The planned time line of the NIFSSWJTU joint project for the CFQS is classified into four phases. In Phase I, physics design, such as magnetic configuration, neoclassical transport property, MHD stability, and engineering design for modular coils, vacuum vessel, supporting structure, power supply for modular coils, etc. are performed. The project is now in Phase I. In Phase II (early 2019 ), fabrication of CFQS and transfer of heating and advanced diagnostic systems from NIFS to SWJTU, such as gyrotron and HIBP, will be performed. In Phase III (early 2021 ), after verification of the accuracy of the CFQS and commissioning are completed, the first plasma of the CFQS will be achieved. In Phase IV, physics experiments will begin.

\section{Acknowledgments}

The authors would like to express their deepest gratitude to Director General Professor Yasuhiko Takeiri of National Institute for Fusion Science, former Vice President of Southwest Jiaotong University Professor Wengui Zhang, and current Vice President of Southwest Jiaotong University Professor Zhongrong Zhou for their strong support and great encouragement of the joint project for the CFQS. This work is performed with the support and under the auspices of the NIFS Collaboration Research Program (NIFS17KBAP034). Also, this work is partly supported by programs of international collaborations with overseas laboratories (UFEX105) and promotion of magnetic confinement research using helical devices in Asia (URSX401). 
[1] Y. Takeiri, IEEE Trans. Plasma Sci. 46, 1141 (2018).

[2] Y. Takeiri, IEEE Trans. Plasma Sci. 46, 2348 (2018).

[3] M. Osakabe et al., IEEE Trans. Plasma Sci. 46, 2324 (2018).

[4] M. Isobe et al., Nucl. Fusion 58, 082004 (2018).

[5] R.C. Wolf et al., Nucl. Fusion 57, 102020 (2017).

[6] T.S. Pedersen et al., Phys. Plasmas 24, 055503 (2017).

[7] J. Nührenberg et al., in Theory of Fusion Plasmas (Proc. Joint Varenna-Lausanne Int. Workshop Varenna, 1994), Editrice Compositori, Bologna 3 (1994).

[8] M. Yokoyama et al., Nucl. Fusion 42, 1094 (2002).

[9] K. Matsuoka et al., Plasma Phys. Report 23, 588 (1997).

[10] S. Okamura et al., J. Plasma Fusion Res. SERIES 3, 73 (2000).

[11] S. Okamura et al., Nucl. Fusion 41, 1865 (2001).

[12] K. Matsuoka et al., Fusion Sci. Technol. 46, 378 (2004).

[13] S. Okamura et al., Nucl. Fusion 44, 575 (2004).

[14] A. Reiman et al., J. Plasma Fusion Res. SERIES 1, 429 (1998).

[15] A. Reiman et al., Plasma Phys. Control. Fusion 41, B273 (1999).

[16] G.H. Neilson et al., Phys. Plasmas 7, 1911 (2000).

[17] M.C. Zarnstorff et al., Plasma Phys. Control. Fusion 43, A237 (2001).

[18] M. Drevlak et al., Contrib. Plasma Phys. 53, 459 (2013).

[19] F. Brochard et al., 41st EPS Conference on Plasma Physics, Berlin, June 23-27, 2014. P2.081.

[20] H. Liu et al., Plasma Fusion Res. 13, 3405067 (2018).

[21] A. Shimizu et al., Plasma Fusion Res. 13, 3403123 (2018).

[22] Y. Xu et al., 27th IAEA Fusion Energy Conference (FEC 2018), Ahmedabad, India, 22-27 October 2018. EX/P5-23.

[23] S. Okamura et al., 45th EPS Conference on Plasma Physics, 2-6 July 2018, Prague, Czech Republic, Vol.42A, P5.1034.

[24] Y. Kinosita et al., The 27th International Toki Confer- ence on Plasma and Fusion Research \& The 13th Asia Pacific Plasma Theory Conference, Ceratopia Toki, Toki-city, Gifu, Japan, November 19-22, 2018. P1-40.

[25] M. Isobe et al., 28th EPS Conference on Controlled Fusion and Plasma Physics, Funchal, 18-22 June 2001, ECA Vol.25A, 761 (2001).

[26] M. Isobe et al., J. Plasma Fusion Res. SERIES 5, 360 (2002).

[27] K.C. Shaing et al., Phys. Fluids B 1, 148 (1989).

[28] S.P. Hirshman and J.C. Whitson, Phys. Fluids 26, 3553 (1983).

[29] V.V. Nemov et al., Phys. Plasmas 6, 4622 (1999).

[30] K. Nishimura et al., Fusion Technol. 17, 86 (1990).

[31] M. Nakata et al., The 27th International Toki Conference on Plasma and Fusion Research \& The 13th Asia Pacific Plasma Theory Conference, Ceratopia Toki, Toki-city, Gifu, Japan, November 19-22, 2018. O-1.

[32] Y. Yoshimura et al., Plasma Fusion Res. 3, S1076 (2008).

[33] N.B. Marushchenko et al., Plasma Fusion Res. 2, S1129 (2007).

[34] S. Okamura et al., Nucl. Fusion 35, 283 (1995).

[35] K. Matsuoka et al., Fusion Eng. Des. 26, 135 (1995).

[36] M. Isobe et al., Fusion Sci. Technol. 50, 229 (2006).

[37] S. Okamura et al., Plasma Phys. Control. Fusion 46, A113 (2004).

[38] T. Minami et al., J. Phys.: Conference Series 123, 012006 (2008).

[39] D.A. Spong et al., Phys. Plasmas 18, 056109 (2011).

[40] K. Ogawa et al., The 27th International Toki Conference on Plasma and Fusion Research \& The 13th Asia Pacific Plasma Theory Conference, Ceratopia Toki, Toki-city, Gifu, Japan, November 19-22, 2018. P1-39.

[41] A. Fujisawa et al., Rev. Sci. Instrum. 67, 3099 (1996).

[42] H. Iguchi, J. Plasma Fusion Res. SERIES 4, 82 (2001).

[43] A. Shimizu et al., Rev. Sci. Instrum. 89, 113507 (2018). 